Review

\title{
Pharmacokinetics, Metabolism and Toxicity of Carbon Nanotubes for Bio- medical Purposes
}

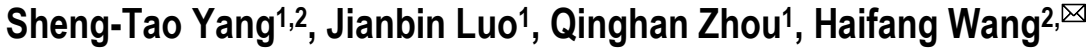 \\ 1. College of Chemistry and Environment Protection Engineering, Southwest University for Nationalities, Chengdu 610041, China. \\ 2. Institute of Nanochemistry and Nanobiology, Shanghai University, Shanghai 200444, China.
}

$\triangle$ Corresponding author: Prof. Haifang Wang, Institute of Nanochemistry and Nanobiology, Shanghai University, Shanghai 200444, China. Tel: 86-21-66135276; E-mail: hwang@shu.edu.cn

(c) Ivyspring International Publisher. This is an open-access article distributed under the terms of the Creative Commons License (http://creativecommons.org/ licenses/by-nc-nd/3.0/). Reproduction is permitted for personal, noncommercial use, provided that the article is in whole, unmodified, and properly cited.

Received: 2011.10.10; Accepted: 2011.11.20; Published: 2012.03.05

\begin{abstract}
Carbon nanotubes (CNTs) have attracted great interest of the nano community and beyond. However, the biomedical applications of CNTs arouse serious concerns for their unknown in vivo consequence, in which the information of pharmacokinetics, metabolism and toxicity of CNTs is essential. In this review, we summarize the updated data of CNTs from the biomedical view. The information shows that surface chemistry is crucial in regulating the in vivo behaviors of CNTs. Among the functionalization methods, PEGylation is the most efficient one to improve the pharmacokinetics and biocompatibility of CNTs. The guiding effects of the pharmacokinetics, metabolism and toxicity information on the biomedical applications of CNTs are discussed.
\end{abstract}

Key words: Carbon nanotubes, pharmacokinetics, metabolism, toxicity, biomedical applications.

\section{Introduction}

Since the discovery, carbon nanotubes (CNTs) have attracted great interest for their potential applications in various areas [1]. In biomedical area, CNTs have been widely used in bio-analysis, therapeutics, drug delivery and early diagnostics. These applications are booming in recent years [2-4]. For example, Moon et al. showed that CNTs could target and destruct tumors by photothermal effect in situ [5]. Liu et al. reported that CNTs could deliver doxorubicin and paclitaxel to tumors to enhance the tumor growth inhibition [6, 7]. The applications of CNTs in Raman imaging [8], near infrared (NIR) fluorescence imaging [9] and radiological imaging [10] were also reported. Moreover, there is a trend to combine the therapeutics and early diagnostics (namely theranostics) together [11-13]. Such combinations on CNTs have been done $[2,14]$.

With the rapid development of CNTs in bio- medical area, the safety concern is aroused and studied among scientists of chemistry, pharmacology and toxicology $[15,16]$. Our group documented that the information on the absorption, distribution, metabolism, excretion/toxicity (ADME/T) of CNTs was indispensible for the safety studies [17]. We unveiled that CNTs were easily trapped in reticuloendothelial system (RES) and induced slight toxicity to liver and lungs [18-20]. Other reports suggested that CNTs were nontoxic after careful surface modification [21, 22]. However, the guiding information for theranostics is still limited to date.

In this review, we summarize the updated data of CNTs from the biomedical view with focus on the pharmacokinetics, metabolism and toxicity of CNTs in vivo. We intend to elucidate the connection between the pharmacological applications and toxicological information of CNTs. The importance of surface 
chemistry in regulating the in vivo behavior of CNTs is stressed.

\section{Pharmacokinetics and biodistribution of CNTs}

The pharmacokinetics and biodistribution of CNTs have been studied as long as the safety issue of CNTs was proposed [23]. The blood circulation time and accumulation of CNTs in animal affect their biomedical applications and toxicity. Serving as a drug vehicle, the long blood-circulated CNTs may accumulate more in tumor, making them efficient for the cancer diagnostics and treatment [24]. To study the pharmacokinetics and biodistribution of CNTs, various analytical techniques have been developed for measuring CNTs in bio-samples [17]. With more information collected, we might get a chance to know how these data guide the biomedical applications of CNTs.

Pristine CNTs are hydrophobic and easy to aggregate. It is difficult to disperse them in biosystems. Studying pristine CNTs is in the pure toxicological evaluations as well as the professional risk assessments. For the biomedical applications, CNTs are dispersed with the aid of suspending reagents or chemical functionalization. Here, we only discuss the pharmacokinetics and biodistribution of these dispersible CNTs.

CNTs, which are simply dispersed with surfactant, are easily cleared from blood circulation and accumulate in RES organs. We studied the biodistribution of Tween 80 dispersed single-walled CNTs (SWCNTs) in mice (Figure 1) [19]. The skeleton of SWCNTs was labeled with stable isotope ${ }^{13} \mathrm{C}$ and the SWCNT contents in tissues were acquired by measuring the ${ }^{13} \mathrm{C}$ abundance. Tween 80 dispersed SWCNTs were fully cleared from blood circulation at

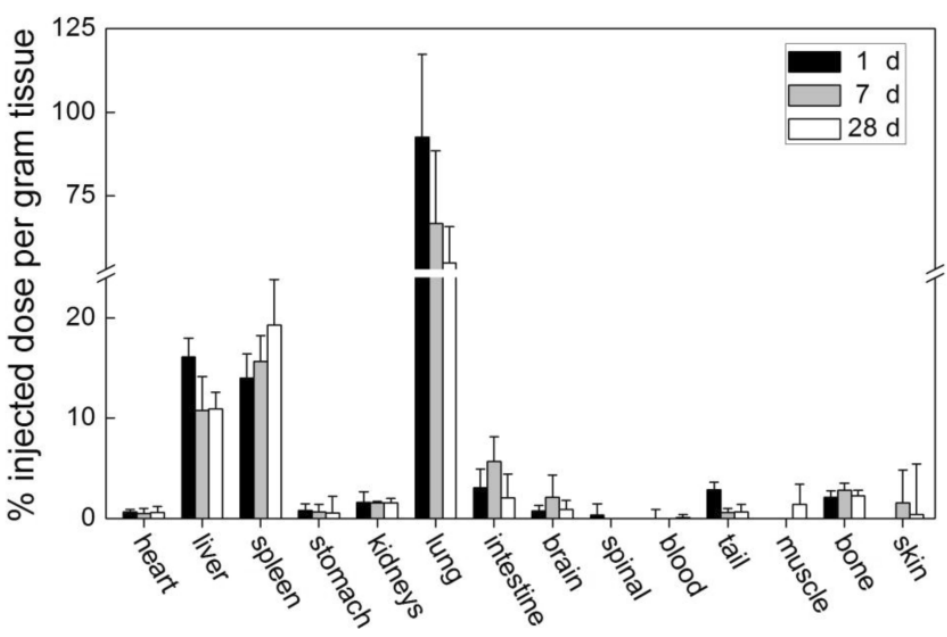

1 day post intravenous injection. They were easily recognized by macrophages and thereby captured by RES in liver and spleen. These SWCNTs remained in liver and spleen for over 3 months [20]. The less dispersed SWCNTs forming larger aggregates were filtered by pulmonary capillary vessels. According to the results of ${ }^{13} \mathrm{C}$ abundance measurements, CNT content in lungs decreased in the first month postexposure. Very similar results were reported by Cherukuri et al. They dispersed SWCNTs with a surfactant named Pluronic F108 and the sample was centrifuged at $100,000 \mathrm{~g}$ for 4 hours to remove the aggregated SWCNTs [25]. By measuring the characteristic NIR fluorescence of individual SWCNTs, they found that Pluronic F108 dispersed SWCNTs majorly accumulated in liver, while the pulmonary uptake was undetectable. Considering only individual SWCNTs emit NIR fluorescence, it is unknown from this case whether SWCNTs aggregate in vivo or not. For multi-walled CNTs (MWCNTs), similar results were reported. Tween 80 dispersed MWCNTs accumulated in liver and spleen with a short blood circulation time [26]. Serum dispersed MWCNTs accumulated in lungs [27]. In a word, the simply dispersed CNTs are easily recognized by macrophages and cleared from blood very fast. This characteristic limits their application in drug delivery. However, simply dispersed CNTs may find their position in diagnostics and thermal therapy. Yang et al. reported that CNTs could be recognized by lymph cells and migrate along lymph vessels [28]. Thus, CNTs may be promising materials for sentinel lymph node detection, which is crucial in cancer therapy. Moreover, the slow migration of CNTs makes them ideal for thermal therapy, because CNTs injected in tumor would stay for a long time. Under NIR irradiation or in radiofrequency field, the heat generated by CNTs could kill tumor cells $[5,29]$.

Figure I. Biodistribution of Tween 80 dispersed SWCNTs in mice after intravenous injection. Adapted from reference [19] with permission. 
By carefully choosing suspending reagents, the pharmacokinetics and biodistribution of CNTs can be improved significantly. Dai and coworkers developed polyethylene glycol-phospholipid (PEG-PL) dispersed CNTs for biomedical detection, imaging, thermal therapy and drug delivery. PEG is one of the most efficient reagents to improve the pharmacokinetics behavior of exotic substances by shielding the exotic substances from the opsonin recognition [30]. PEG chains were linked to PL molecules to form a hydrophilic-hydrophobic structure [24, 31]. PEG-PL suspended CNTs undergo hydrophobic interaction between PL and CNTs. PEG-PL prolonged the blood circulation half-life and reduced the RES capture of SWCNTs in vivo. Liu et al. reported that $\mathrm{PEG}_{2000}-\mathrm{PL} / \mathrm{SWCNTS}$ (PEG-PL dispersed SWCNTs; the molecule weight of PEG: 2000) had a half-life of 1.2 hours [24]. The half-life could be further prolonged by using longer PEG chains and even branched PEG chains. The circulation half-life of PEG5000-PL/SWCNTs increased to about 5 hours [31]. Using branched PEG chains, the value could be higher than 15 hours. More recently, Prencipe et al. used
үPGA-Py-mPEG [poly-(Y-glutamic acid)-pyrine(30\%)-poly (ethylene glycol) methyl ethers) $(70 \%)]$ to disperse SWCNTs and studied their blood circulation half-life [32]. The dispersion was very stable and prolonged the circulation half-life of SWCNTs to 22.1 hours. Accordingly, the RES capture decreased along with the change of PEG-PL molecules. The hepatic uptake of $\mathrm{PEG}_{2000} \mathrm{PL} / \mathrm{SWCNT}$ s was $70 \% \mathrm{ID} / \mathrm{g}$ (\% injected dose per gram tissue), which decreased to $40 \% \mathrm{ID} / \mathrm{g}$ for $\mathrm{PEG}_{5000} \mathrm{PL} / \mathrm{SWCNT}$ and $30 \% \mathrm{ID} / \mathrm{g}$ for br-PEG7000-PL/SWCNTs (br: branched). The PEG density on SWCNTs also regulated the blood circulation, biodistribution and tumor uptake of SWCNTs. The blood circulation half-life increased along with increasing the PEG density, while the RES uptake decreased accordingly [33]. The good behaviors of PEG-PL/SWCNTs in vivo make them very useful in biomedical areas. PEG-PL/SWCNTs have been used to target tumors and deliver doxorubicin and paclitaxel for cancer therapy [6,7]. They have also been used for photothermal ablation of tumors [33]. In addition, they have been applied in Raman and photoacoustic imaging for diagnostics [10,34].
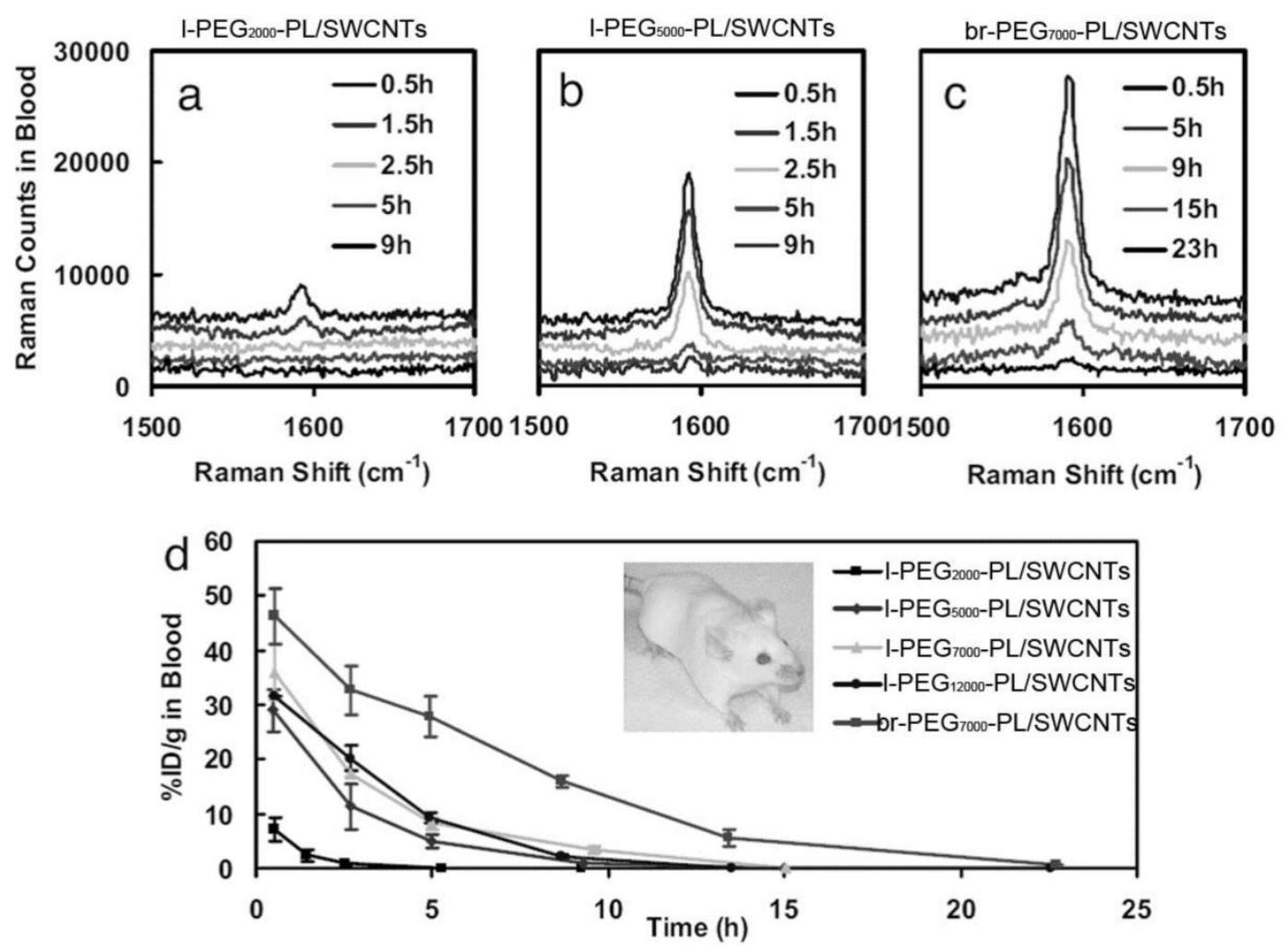

Figure 2. Pharmacokinetics of PEG-PL/SWCNTs in mice after intravenous injection. (a) Raman spectra of blood samples after the injection of I-PEG $2000-P L / S W C N T s$ (I: linear); (b) Raman spectra of blood samples after the injection of I-PEG5000-PL/SWCNTs; (c) Raman spectra of blood samples after the injection of br-PEG7000-PL/SWCNTs; (d) PEG-PL/SWCNTs concentrations in blood at different time points postexposure. Adapted from reference [3I] with permission. 
The studies of other nanoparticles suggest that covalent PEGylation is more efficient than noncovalent PEGylation [30]. This is true for CNTs, too. We performed a pharmacokinetics evaluation of PEG-SWCNTs (covalently PEGylated SWCNTs) in mice [35]. After $\mathrm{PEG}_{1500}$ was covalently linked to the carboxyl groups of SWCNTs, the blood circulation half-life of SWCNTs increased to 15.3 hours. Even short PEG chains can prolong the circulation half-life of CNTs efficiently while using covalent PEGylation. Correspondingly, the RES capture of

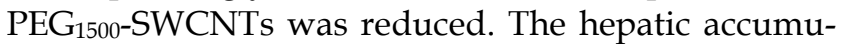
lation level was $19.1 \% \mathrm{ID} / \mathrm{g}$ for $\mathrm{PEG}_{1500}-\mathrm{SWCNTs}$. The good pharmacokinetics profile of PEG-SWCNTs makes them promising in biomedical applications. However, the biomedical applications of PEG-SWCNTs are fewer than those of PEG-PL/SWCNTs.
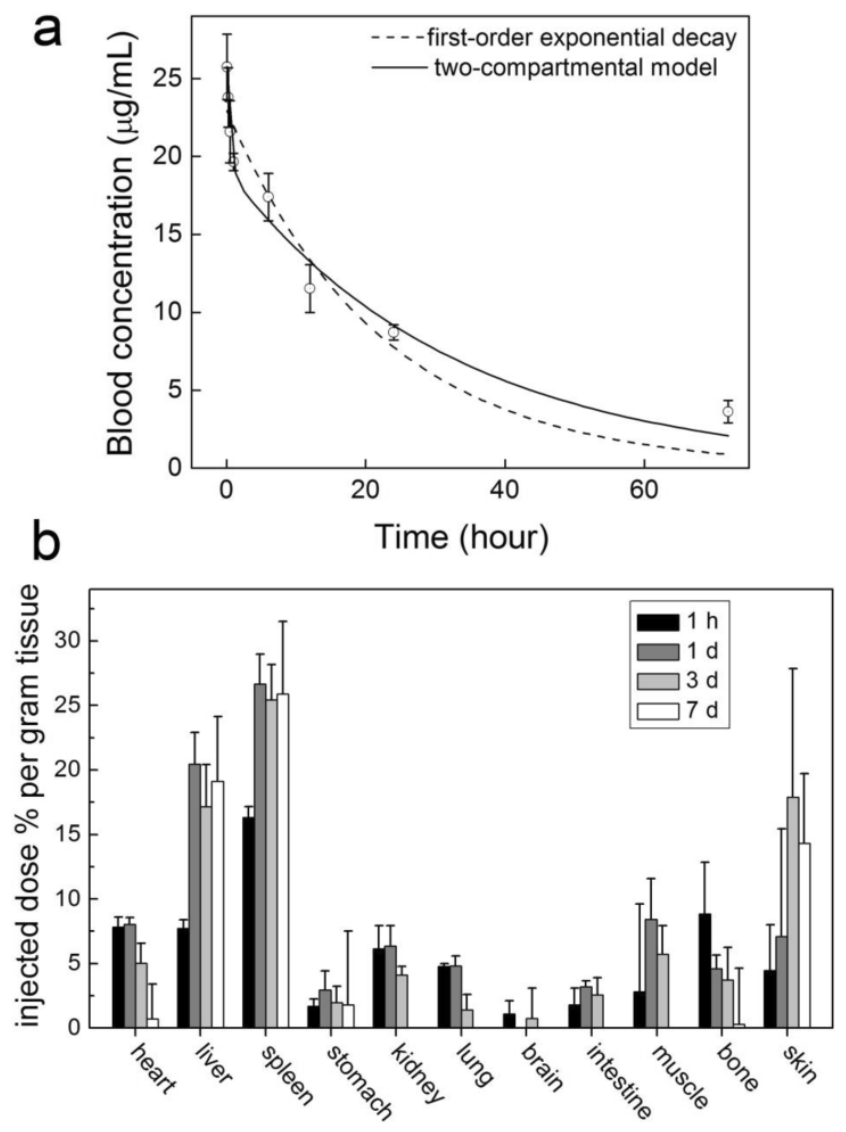

Figure 3. Time-dependent PEG-SWCNT concentrations in blood (a) and mice (b) after the intravenous injection to mice. Adapted from reference [35] with permission.
There might be two reasons: Firstly, the synthesis of PEG-SWCNTs is much more difficult than that of PEG-PL/SWCNTs, which limits the involvement of more researchers. Secondly, covalent PEGylation destroys the intact nature of SWCNTs, making some unique properties of SWCNTs lost. After covalent PEGylation, SWCNTs have strong fluorescence, which hides the Raman signal of SWCNTs [36]. The covalent functionalization also destroys the NIR fluorescence signal of SWCNTs. Still, drug delivery systems based on PEG-CNTs have been developed at cellular level [37, 38] and in vivo level [39].

Another kind of functionalized CNTs was developed by Prato and coworkers [40]. CNTs were functionalized via cycloaddition reactions, where functional groups were directly attached to the carbon skeleton of CNTs. Singh et al. reported the pharmacokinetics and biodistribution of amino-CNTs [41]. Amino-CNTs were removed from body very quickly. A small amount of injected amino-CNTs were trapped in kidneys $(20 \% \mathrm{ID} / \mathrm{g})$, skin $(9 \% \mathrm{ID} / \mathrm{g})$, muscle $(8.5 \% \mathrm{ID} / \mathrm{g})$ and lungs $(1.3 \% \mathrm{ID} / \mathrm{g})$. At 3 hours post injection, only $2 \%$ ID of amino-CNTs were detected in body. Another evaluation was performed by McDevitt et al. [42, 43]. They reported that amino-SWCNTs remained in body for more than 24 hours. At 3 hours postexposure, amino-SWCNTs accumulated in liver $(17.8 \% \mathrm{ID} / \mathrm{g})$, spleen $(14.3 \% \mathrm{ID} / \mathrm{g})$, kidneys $(8.3 \% \mathrm{ID} / \mathrm{g})$ and skin $(2.3 \% \mathrm{ID} / \mathrm{g})$. Except in kidneys, amino-SWCNT contents kept unchanged after 24 hours. The different CNT sources and synthesis conditions might attribute to the contradictory results of the two groups. Such differences would lead to different CNT lengths, functionalization degree and so on. Due to the fast clearance of amino-CNTs from blood, the in vivo applications of amino-CNTs still require more efforts to improve their pharmacokinetics profile, though various demonstrations have been established at cellular level [44, 45]. However, the amino-CNTs showed potential in delivering siRNA to tumor via intratumoral injection [46] and to brain via injection in the primary motor cortex [47]. For future studies, prolonging the side chains of amino functional groups might be helpful to improve the pharmacokinetics.

Other CNT samples tested show less potential in biomedical areas. We have studied the pharmacokinetics of hydroxylated SWCNTs (SWCNTols) [23, 48] and taurine functionalized MWCNTs (tau-MWCNTs) [18]. SWCNTols distributed rapidly throughout the whole body at 2 min postexposure to mice via tail intravenous injection. The blood circulation half-life of SWCNTols was around $10 \mathrm{~min}$ [48]. The migration of SWCNTols in body seemed nearly free, that was 
also evidenced by very similar distribution profiles after the exposures by different injection methods [23]. The very short circulation time and free migration will limit the bio-applications of SWCNTols. On the other hand, tau-MWCNTs targeted liver quickly and stayed long there for months. The hepatic uptake of tau-MWCNTs was higher than $80 \%$ ID (\% of injected dose) $[18,26]$, while the circulation half-life was around $12 \mathrm{~min}$ [26]. The short circulation time and high RES uptake indicate the difficulty of the bioapplications of tau-MWCNTs. Tyrosine functionalized MWCNTs suffer the same problem as tau-MWCNTs do [49].
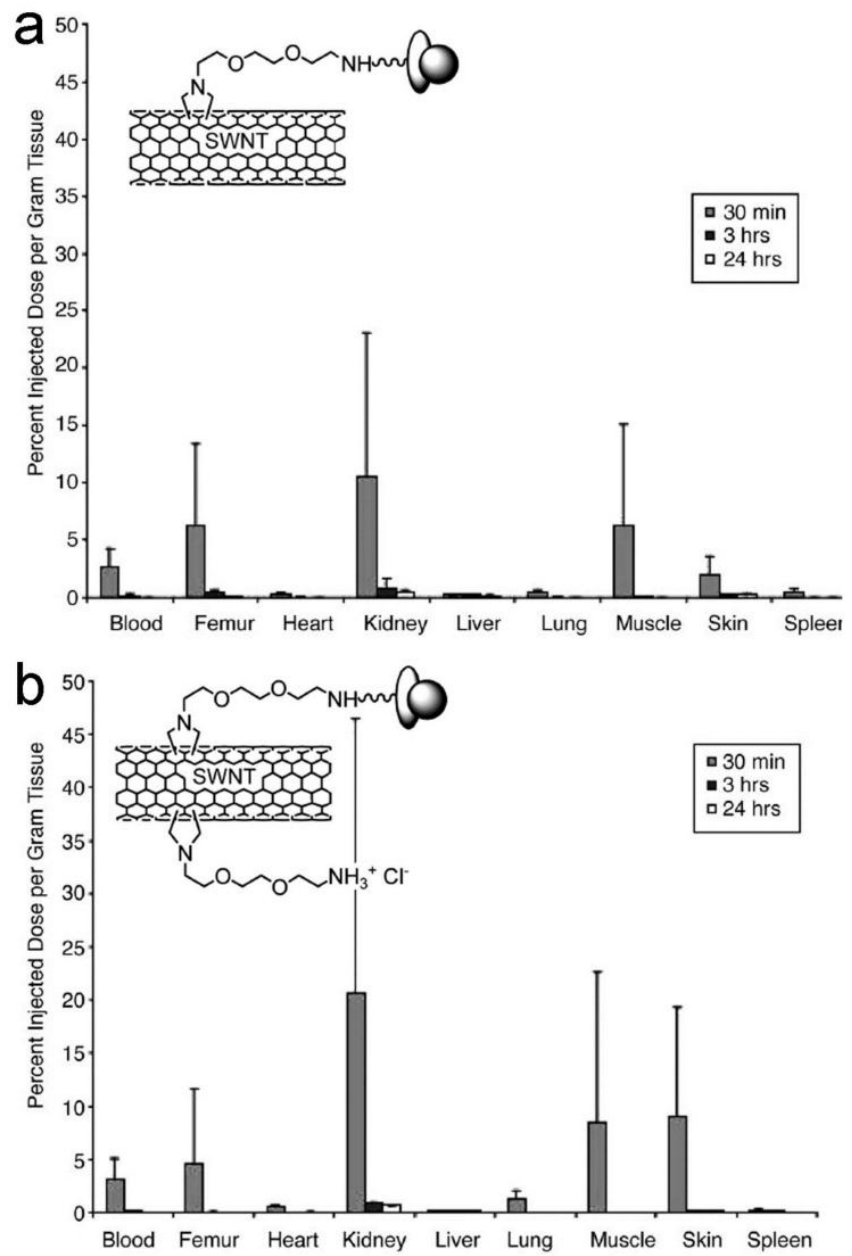

Figure 4. Biodistribution of two different amino-SWCNTs in mice after intravenous injection. Insets show the schematic structures of amino-SWCNTs. Adapted from reference [4I] with permission.

As discussed above, the pharmacokinetics of CNTs is very important for the biomedical applications. There are many parameters regulating the pharmacokinetics of CNTs, such as size, charge and surface chemistry. For CNTs, when the length is smaller than $2 \mu \mathrm{m}, \mathrm{CNTs}$ can escape the pulmonary capillary vessels and their pharmacokinetics is mainly determined by the surface chemistry. Among the reported surface chemistry, PEGylation is a most efficient method in improving the pharmacokinetics profile of CNTs. The development of easy, cheap and reproducible PEGylation techniques becomes a crucial route for the biomedical applications of CNTs.

\section{Metabolism and transformation of CNTs}

CNTs employed for biomedical purposes are in forms of either dispersed or functionalized ones. After introducing to biosystems, it is possible that these CNTs are metabolized into other substances. The metabolism of CNTs definitely affects their biomedical applications and also might lead to unwanted toxicity. Therefore, the in vivo metabolism of CNTs should be highly concerned. Unfortunately, the metabolism of carbon nanomaterials is very hard to study, because of the lack of proper analytical techniques. Only few pilot studies are available in literature.

The skeleton of CNTs is relatively more stable than their functional groups. We have shown the transmission electron microscopy (TEM) images of SWCNTs and MWCNTs (both pristine and functionalized) trapped in vivo for more than 4 weeks. The trapped CNTs even stayed as long as 3 months [18, 20]. The typical tube like or linear structure of CNTs was clearly recognized under TEM, suggesting that the carbon skeletons of CNTs were very stable against in vivo metabolism as well as the subsequent acid treatment for preparing the TEM samples. During the purification and shortening processes, CNTs would go through strong acid $\left(\mathrm{HNO}_{3}, \mathrm{HCl}\right.$ and $\left.\mathrm{H}_{2} \mathrm{SO}_{4}\right)$ treatment, sometimes assisted by ultrasonication. Under such harsh conditions, the carbon skeleton only breaks at the injured defects. The stability of CNTs is also reflected by the long-term accumulation in vivo without being metabolized, where CNTs were visualized in mouse organs upon TEM at several months postexposure [18, 20, 31]. Unlike most common results, Allen et al. observed the biodegradation of SWCNTs through enzymatic catalysis in vitro [50]. SWCNTs were incubated in PBS ( $\mathrm{pH}$ 7.0) in presence of horseradish peroxidase and $\mathrm{H}_{2} \mathrm{O}_{2}$ in dark. After 16 weeks degradation, the loss of tube structure was identified by TEM, thermogravimetric analysis (TGA), matrix-assisted laser desorption-ionization time-of-flight mass spectrometry (MOLDI-TOF MS), dynamic light scattering (DLS), nanotube density of states band diagram and UV-vis-NIR spectra. For the 
studies of biocompatibility and applications, the biodegradation of CNTs is an important factor to be regarded. Kagan et al. reported that the degradation by neutrophil myeloperoxidase reduced the pulmonary toxicity of CNTs significantly [51]. The stability of carbon skeleton also implies that CNTs are hard to be metabolized to small molecules, which may easily be excreted via urine and feces. More attention should be paid to design proper surface property of CNTs to accelerate the beneficial excretion of CNTs.

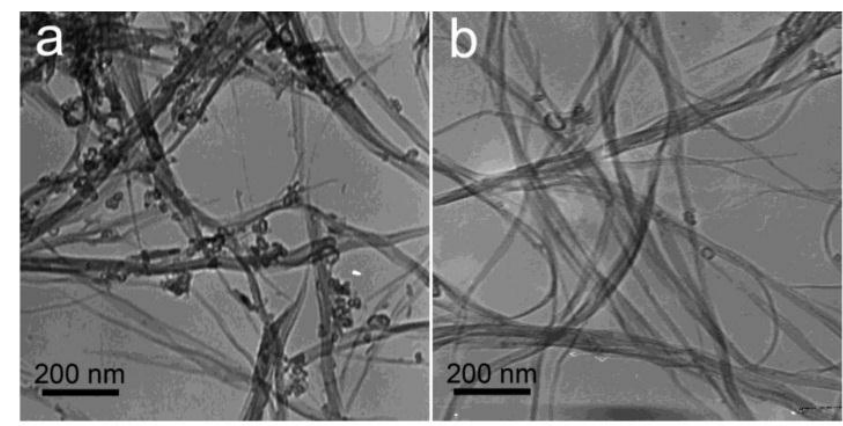

Figure 5. TEM images of SWCNTs in digested solution of liver (a) and lungs (b) at 90 days postexposure. Adapted from reference [20] with permission.

In contrast to the stability of carbon skeleton, the functional groups on CNTs are much easier to fall off from CNTs. There are two kinds of strategies to functionalize CNTs, namely chemical functionalization and dispersion (non-covalent functionalization). Current results suggest that both kinds of functional groups could be detached from CNTs in vivo and this makes functionalized CNTs transformed into less functionalized or pristine CNTs.

CNTs could be non-covalently functionalized by adsorbing reagents to their surface via hydrophobic interaction and п-п interaction. The metabolism of non-covalently functionalized CNTs is generally regarded as desorption of the suspending reagents. Cherukuri et al. studied the replacement of adsorbed surfactants (Pluronic F108) on CNTs by serum proteins using NIR fluorescence [25]. The NIR fluorescence spectrum of SWCNTs broadened and red-shifted after the sample was introduced into the blood circulation. Such phenomenon was also observed after adding the serum proteins. The positions and widths of SWCNT fluorescence peaks varied with the nature of the surrounding medium. Therefore, the serum proteins might replace Pluronic F108 molecules and stick to the surface of SWCNTs. The replacement of adsorbed surfactants by proteins seems to happen only at the high protein concentrations, because at the low protein concentrations, surfactant adsorption is very stable against being replaced. Another interesting example of desorption comes from lysophophatidylcholine (LPC) coated SWCNTs [52]. Roberts et al. reported that LPC coated SWCNTs could be ingested by Daphnia magna. When food supply was stopped, Daphnia magna would take LPC from SWCNT surface as food source. After desorption of LPC, the naked SWCNTs aggregated and were excreted. In contrary to the results of Cherukuri et al. and Roberts et al., Liu et al. found PEG-PL suspended SWCNTs, a novel platform for theranostics, were very stable. The hydrophobic chains of PL stuck to the SWCNT surface, while PEG chain stretched into the aqueous environment. The whole structure was similar to the micelle structure. The suspension was stable against salts, high temperature and also the biosystems [24]. The PEG-PL suspended SWCNTs were excreted via urine and feces [31]. Comparing to the long-term accumulation of pristine SWCNTs, the slow excretion of PEG-PL/SWCNTs implies that such materials are stable in vivo (naked SWCNTs are hardly excreted). The inconsistency in literature should be attributed to the different CNT samples, suspending reagents, and biosystems. In particular, SWCNTs used for the preparation of PEG-PL/SWCNTs were very short ( 100 $\mathrm{nm}$ in length), so the slow excretion might be expected. For theranostics applications, stable suspended CNTs are more preferable. Thus, more efficient and stable suspending reagents, such as PEG-PL, are recommended.

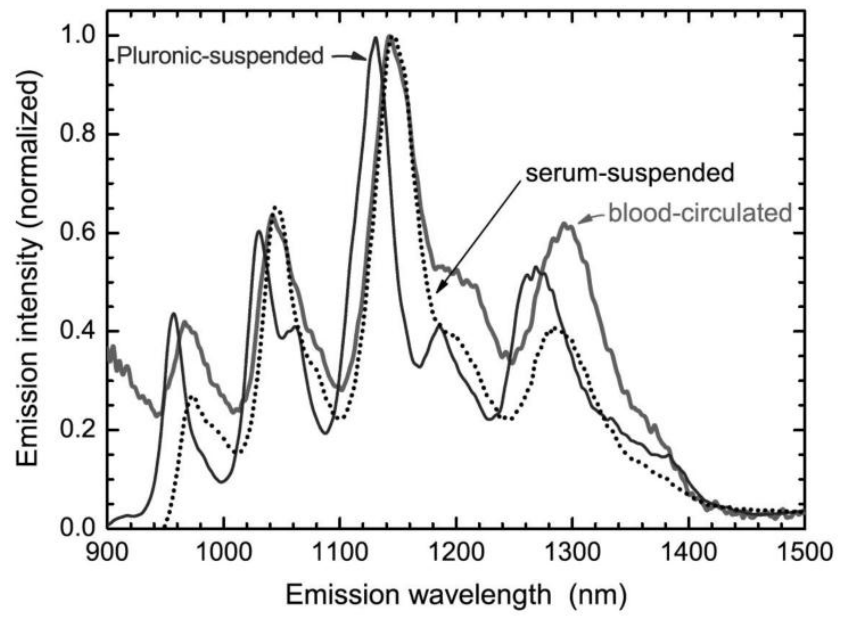

Figure 6. Replacement of Pluronic FI08 on SWCNTs by serum proteins. The desorption is characterized by NIR fluorescence spectra. Adapted from reference [25] with permission. 
Chemical modification is successfully used in the biomedical applications of CNTs. Various molecules, including small molecules, polymers and biomolecules, have been covalently linked to the carbon skeleton of CNTs [2]. Generally, covalent bonds are more stable than non-covalent ones. Therefore, the stability of covalently functionalized CNTs is expected. We performed an in vivo evaluation of PEG-SWCNTs by using Raman spectroscopy [53]. PEGylation made SWCNTs fluorescent, and the Raman signals of SWCNTs were covered by the strong fluorescence. The recovered Raman signals were taken as the indicator of metabolism, because the defunctionalization of PEG-SWCNTs in this case led to the depression of fluorescence and the recovery of Raman signal. After intravenous injection PEG-SWCNTs accumulated in liver and spleen for a long time. Due to the different functions of liver and spleen, PEG-SWCNTs showed different stabilities in these two organs. Liver is the most important metabolism organ, containing plenty of metabolic enzymes. PEG-SWCNTs trapped in liver defunctionalized slowly postexposure. Obvious Raman signal recovery was observed at 4 weeks postexposure. The simulated experiments suggested that the hepatic defunctionalization of PEG-SWCNTs was resulted from both effects of radical attack and acid hydrolysis. On the other hand, PEG-SWCNTs were very stable against biotransformation in spleen for more than 8 weeks. Considering that spleen is the biggest immunological organ and plays important roles in blood purification, the stability of PEG-SWCNTs in spleen is reasonable.

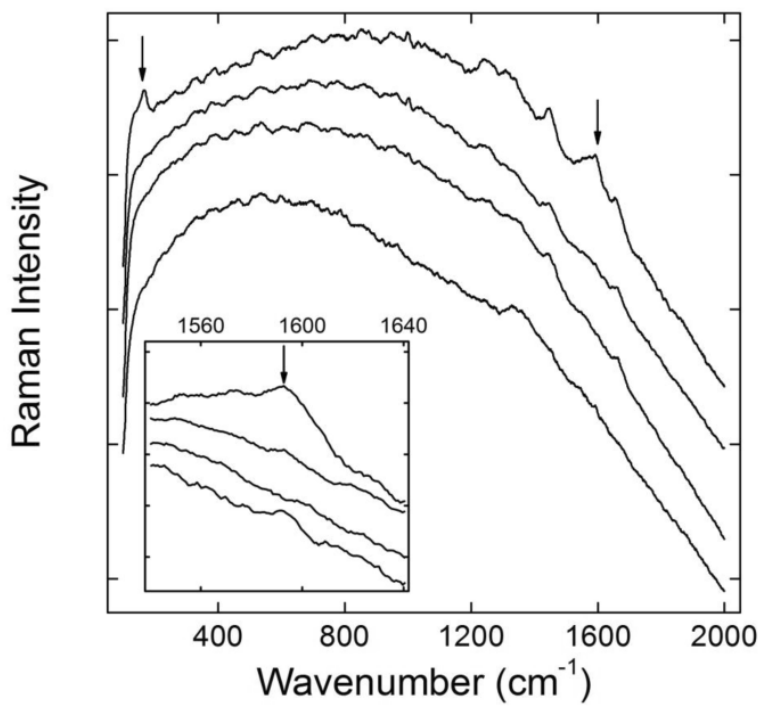

Figure 7. Defunctionalization of PEG-SWCNTs in liver after intravenous injection reflected by Raman spectra (the inset featuring the region around $1590 \mathrm{~cm}^{-1}$, from bottom up: the control, I day, I week, and 4 weeks postexposure). Adapted from reference [53] with permission.
Our results suggest that the metabolism of CNTs in vivo is organ-dependent, which requires individual evaluation for each accumulation organ. The hepatic defunctionalization indicates that liver is the most possible metabolic organ in body for CNTs. Less functionalized CNTs are believed to be more toxic. Therefore, the hepatic toxicity of CNTs needs more attentions.

Because of the high stability of carbon skeleton, the metabolism of CNTs mainly means defunctionalization of the surface functional groups. Comparing the stability of non-covalently and covalently functionalized CNTs in vivo, the covalently functionalized CNTs seem more stable than non-covalently suspended ones. This phenomenon indicates that covalently modified CNTs are more suitable for biomedical use in vivo from the stability view. For the non-covalently suspended CNTs, using more stable suspending reagents, such as PEG-PL is preferred.

\section{Toxicity of CNTs in vivo}

The biosafety of CNTs is currently a most concerned and discussed issue for their biomedical applications. The unique characteristics of CNTs make some traditional toxicological assays inapplicable. For example, Worle-Knirsch et al. reported that MTT (3-(4,5-dimethylthiazol-2-yl)-2,5-diphenyltetrazolium bromide) assay gave false positive results in evaluating the cytotoxicity of CNTs [54]. Toxicity of CNTs remains in debate at cellular level, mainly due to the difference among the CNT samples. At in vivo level, more evaluations are made for the pulmonary toxicity of CNTs, regarding to the professional exposure. For biomedical applications, toxicological information achieved through intravenous, subcutaneous and gastrointestinal exposure is more important. Current results conclude that CNTs are of low toxicity through the aforementioned pathways.

CNTs show relative high biocompatibility post intravenous injection. Generally, simply dispersed CNTs are more toxic than the covalently modified ones, but the toxicity of simply dispersed CNTs is still very low, even with a long-term observation. We evaluated the long-term toxicity of Tween 80 dispersed SWCNTs [20]. Serum biochemical index indicated that SWCNTs induced damage to hepatic function. The dose-dependent increases of alanine aminotransferase (ALT) and aspartate aminotransferase (AST) levels reminded us that there was dose effect for the toxicity of SWCNTs. Histopathological analyses showed that SWCNTs induced inflammation and inflammatory cell infiltration in lungs. We did not find the changes of serum immunological levels and the increase of apoptosis. The toxicity of SWCNTs was 
closely related to the oxidative stress. Lacerda et al. reported that pristine MWCNTs dispersed by serum were more toxic than amino-MWCNTs [27]. More weight loss and inflammation occurred in the pristine MWCNTs treated mice.
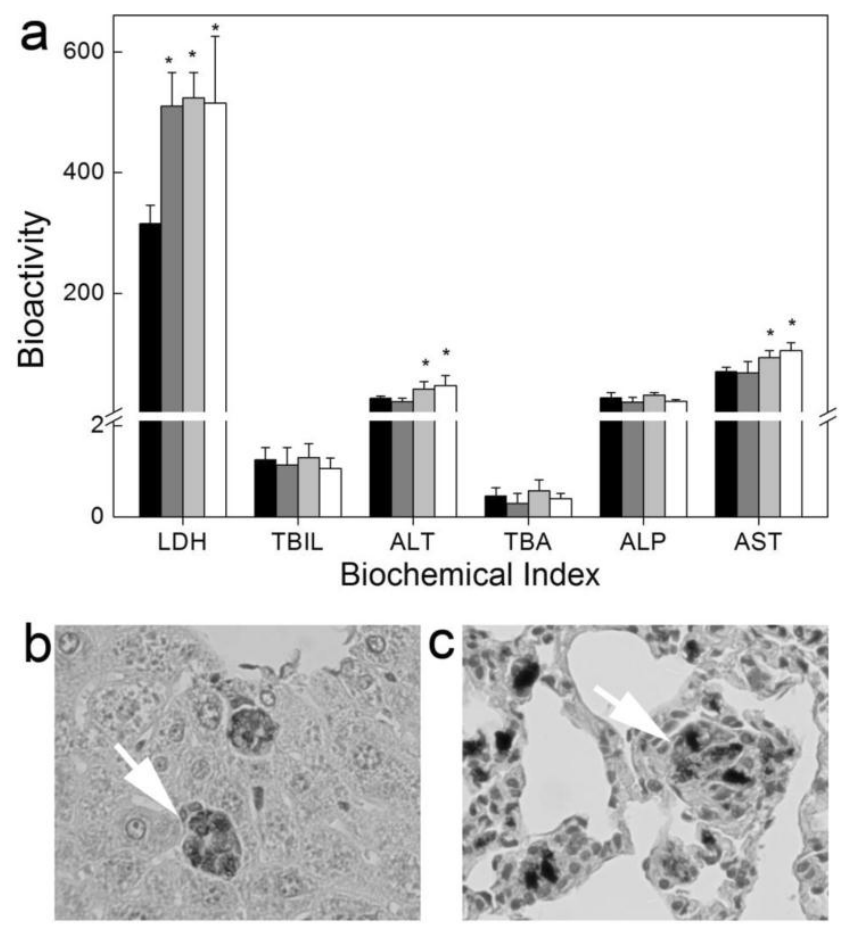

Figure 8. Long-term toxicity of Tween 80 dispersed SWCNTs to mice after intravenous injection. (a) Serum biochemical index; (b, c) Histopathology of liver (b) and lungs (c). White arrows indicate the trapped SWCNTs. Adapted from reference [20] with permission.

Chemically functionalized CNTs show higher biocompatibility. We performed the first toxicity evaluation of CNTs after intravenous injection [18]. The serum biochemical index and histopathological characteristics of mice kept unchanged after exposure to tau-MWCNTs. We concluded that tau-MWCNTs were nearly nontoxic. Generally, other chemically functionalized CNTs are also of low toxicity. For example, Deng et al. reported that oxidized MWCNTs (O-MWCNTs) and tau-MWCNTs induced low toxicity to mice [55]. Only slight inflammatory response, mitochondria destruction and oxidative damage were observed at high concentrations. Qu et al. reported that less dispersed O-MWCNTs were trapped in lungs and induced inflammation there [56]. O-MWCNTs did not affect the hepatic function. However, slight histopathological changes presented in spleen and kidneys. Repeated administrations of O-MWCNTs and MWCNTs-NH $\mathrm{N}_{2}(\mathrm{O}-\mathrm{MWCNT}$ linked with 1, 3-diaminopropane) to mice induced decreased germinative layer thickness and vacuolization of Sertoli cells [57]. The testis damage was reversible and the fertility was not affected at all. Other indicators, such as oxidative stress, bodyweight, organ index, sperm concentration, motility, morphology and acrosome integrity, kept at the normal levels just as the control group. Amino-MWCNTs also showed very good biocompatibility [27]. They did not accumulate long in body, and the toxicological indicators (histopathology, serum biochemistry, hematology and urinalysis) remained normal. It seems that well functionalization improves the dispersibility of CNTs, avoids the filtration of CNTs by pulmonary capillary vessels and reduces the toxicity consequently.

Again, PEGylated CNTs exhibit very good biocompatibility. Schipper et al. reported that PEG-SWCNTs and PEG-PL/SWCNTs were nontoxic and biocompatible [22]. Although both SWCNTs retained in liver for more than 4 months, mice exposed to PEG-SWCNTs and PEG-PL/SWCNTs behaved normally. There was no statistical difference of body weight increase and blood pressure between the exposed and the control groups. The hematological and serum biochemical indicators also suggested that both samples were nontoxic. Slightly higher toxicity of PEG-MWCNTs (covalently PEGylated MWCNTs) was detected and reported by Zhang et al. [58]. Inflammatory infiltration and low-grade spotty necrosis presented in histopathological analyses. The damage to mitochondria, such as mitochondrial destruction and lysis of mitochondrial crest, were observed under TEM. Inflammatory gene expression was up-regulated, but tumor necrosis factor-a (TNF-a) level remained unchanged. The hepatic function was not affected according to serum biochemical indicators. No oxidative damage was detected, because glutathione (GSH) and superoxide dismutase (SOD) remained normal levels. It is worth to note that PEG-MWCNTs only showed toxicity at very high dosage $(60 \mathrm{mg} / \mathrm{kg})$. At lower dosages, PEG-MWCNTs were nearly nontoxic. The nontoxic nature of PEGylated CNTs at low dosages is a very good property for biomedical applications. Considering that there might be metabolism (defunctionalization) in vivo, the fate and consequence of PEGylated CNTs should be carefully studied in future.

CNTs exposed by oral gavage are of low toxicity, too. Folkmann et al. reported that SWCNTs induced oxidative damages to DNA after oral gavage [59]. The indicator, premutagenic 8-oxo-7,8-dihydro-2'deoxyguanosine (8-oxodG), only increased in liver 
and lungs. SWCNTs suspended in corn oil induced more DNA damages than these in PBS. Yang et al. observed the oxidative damage in mice induced by SWCNTs via oral gavage [60]. SWCNTs were found in liver and intestine. SWCNTs targeted lysosome and mitochondria, and then induced collapse of mitochondrial membrane potentials, generating more reactive oxygen species leading to the mitochondrial damage.

CNTs can be used as tissue engineering materials for cell growth. After implanting subcutaneously,
CNTs exhibited very good biocompatibility. Bellucci et al. reported that implanted MWCNT paper did not arouse any serious toxicity, except very limited inflammation [61]. Fraczek et al. found that implanted SWCNTs and MWCNTs induced inflammation [62]. Smaller SWCNT aggregates migrated to lymph nodes nearby. Other reports on the toxicity of CNTs to skin suggested that CNTs were biocompatible to skin, which was consistent with the good biocompatibility after subcutaneously planting.
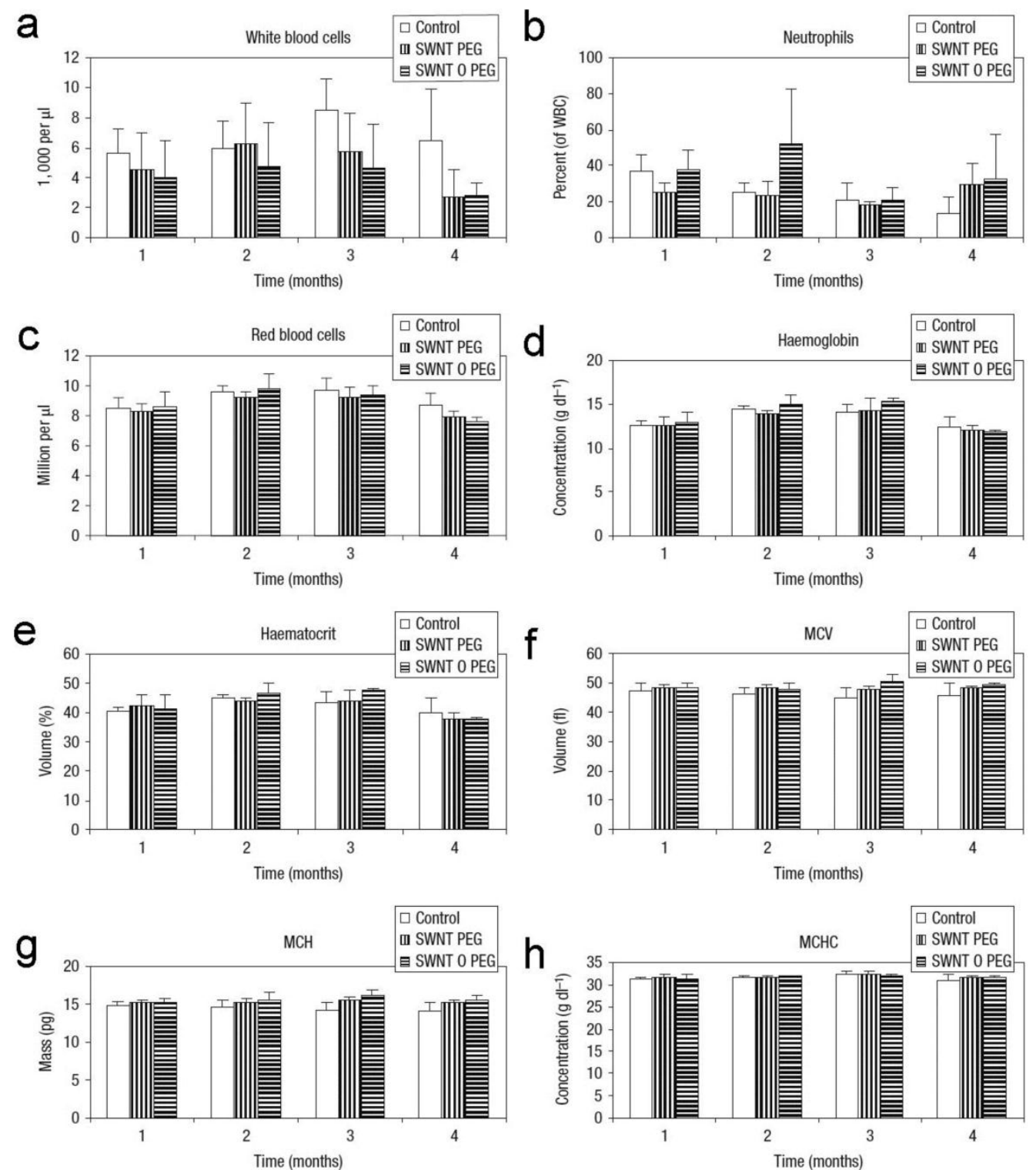

Figure 9. Hematological indicators of mice exposed to PEG-SWCNTs and PEG-PL/SWCNTs. MCV: mean corpuscular volume; MCH: mean corpuscular hemoglobin; MCHC: mean corpuscular hemoglobin concentration. Adapted from reference [22] with permission. 

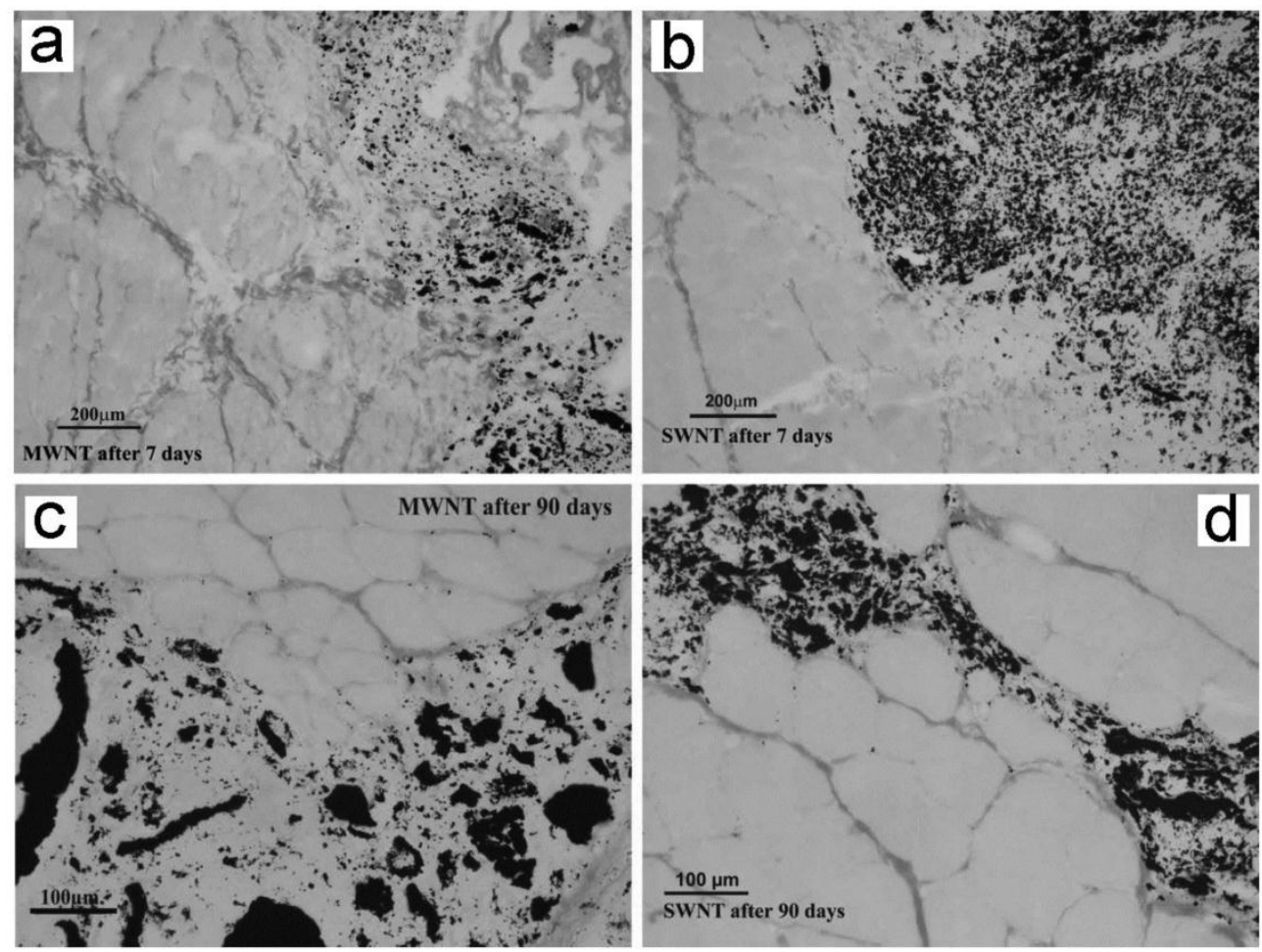

Figure 10. Muscle implanted with CNTs after 7 and 90 days of implantation. No uniform fibrous capsule separates the implant from the muscle tissue. Adapted from reference [62] with permission.

As discussed above, there are several parameters affecting the toxicity of CNTs in vivo. Oxidative stress is observed in despite of the administration pathways. Metal impurities might contribute partially to the oxidative stress, thus, careful purification of CNTs is necessary. In fact, the surface chemical functionalization is also a purification process for CNTs. Among these functionalization methods, PEGylation is highly recommended. A possible hypothesis is that PEGylation prevents proteins adsorb onto CNTs, hence reduces the interaction between CNTs and biosystem. In addition to surface chemistry, other parameters might also influence the toxicity of CNTs. Shape is an important factor influencing the toxicity of CNTs. In literature, the fiber-like CNTs (longer) were more toxic than the rod-like CNTs (shorter) [63]. Size is another important influencing factor. Bigger aggregates of CNTs accumulate in lungs and induce the pulmonary toxicity, while smaller ones hardly affect the pulmonary functions [20,27].

The available safety data collectively indicate that CNTs are of low toxicity via various exposure pathways for biomedical applications. CNTs induce meaningful toxicity only when a very high dosage is administrated. Thus, we come to a verdict that CNTs are generally biocompatible and low toxic for the biomedical purposes. More toxicity evaluations are encouraged to give the safety threshold value (dosage limit) of different CNTs and clarify the toxicological mechanism. Carefully optimizing the physicochemical parameters to minimize the toxicity of CNTs is highly favorable.

\section{Conclusions}

In summary, we summarized the updated data of CNTs from the biomedical view with focus on the pharmacokinetics, metabolism and toxicity of CNTs in vivo. The connection between the pharmacological applications and toxicological information of CNTs was discussed. The pharmacokinetics, metabolism and toxicity of CNTs are dependent on the physicochemical parameters of CNTs and regulated by the surface chemistry. Well functionalized CNTs by both non-covalent and covalent methods are promising for the biomedical applications. PEGylation is a most efficient method to improve the in vivo behaviors of CNTs. In future studies, systematic evaluation of the long-term toxicity of CNT exposure is worthy of special attention. Moreover, establishing fast and reliable analytical techniques for in vivo behavior evaluation should always be concerned, particularly, for the newly developed nanomaterials. 


\section{Acknowledgement}

We thank Prof. Yuanfang Liu at Peking University for critical reading of the manuscript. We acknowledge financial support from the China Ministry of Science and Technology (973 Project No. 2011CB933402), the China Natural Science Foundation (No. 21071094), Shanghai Municipal Education Committee (11ZZ82), Key Project of Chinese Ministry of Education (211053), the Fundamental Research Funds for the Central Universities, Southwest University for Nationalities (No. 11NZYBS06).

\section{Conflict of Interest}

The authors have declared that no conflict of interest exists.

\section{References}

1. Schnorr JM, Swager TM. Emerging applications of carbon nanotubes. Chem Mater 2011; 23: 646-657.

2. Lu F, Gu L, Meziani MJ, et al. Advances in bioapplications of carbon nanotubes. Adv Mater 2009; 21: 139-152.

3. Thakare VS, Das M, Jain AK, et al. Carbon nanotubes in cancer theragnosis. Nanomedicine 2010; 5: 1277-1301.

4. Vashist SK, Zheng D, Pastorin G, et al. Delivery of drugs and biomolecules using carbon nanotubes. Carbon 2011; 49:4077-4097.

5. Moon HK, Lee SH, Choi HC. In vivo near-infrared mediated tumor destruction by photothermal effect of carbon nanotubes. ACS Nano 2009; 3: 3707-3713.

6. Liu Z, Chen K, Davis C, et al. Drug delivery with carbon nanotubes for in vivo cancer treatment. Cancer Res 2008; 68: 6652-6660.

7. Liu Z, Fan AC, Rakhra K, et al. Supramolecular stacking of doxorubicin on carbon nanotubes for in vivo cancer therapy. Angew Chem Int Ed 2009; 48: 7668-7672.

8. Keren S, Zavaleta C, Cheng Z, et al. Noninvasive molecular imaging of small living subjects using Raman spectroscopy. Proc Natl Acad Sci USA 2008; 105: 5844-5849.

9. Welsher K, Sherlock SP, Dai H. Deep-tissue anatomical imaging of mice using carbon nanotube fluorophores in the second near-infrared window. Proc Natl Acad Sci USA 2011; 108: 8943-8948.

10. Hong SY, Tobias G, Al-Jamal KT, et al. Filled and glycosylated carbon nanotubes for in vivo radioemitter localization and imaging. Nat Mater 2010; 9: 485-490.

11. Ye $Y, C$ Chen $X$. Integrin targeting for tumor optical imaging. Theranostics 2011; 1:102-126.

12. Huang $\mathrm{P}, \mathrm{Xu} \mathrm{C}$, Lin J, et al. Folic acid-conjugated graphene oxide loaded with photosensitizers for targeting photodynamic therapy. Theranostics 2011; 1:240-250.

13. Lukianova-Hleb EY, Oginsky AO, Samaniego AP, et al. Tunable plasmonic nanoprobes for theranostics of prostate cancer. Theranostics 2011; 1:3-17.

14. Bhirde AA, Liu G, Jin A, et al. Combining portable Raman probes with nanotubes for theranostic applications. Theranostics 2011; 1:310-321.

15. Wick P, Clift MJD, Rosslein M, et al. A brief summary of carbon nanotubes science and technology: A health and safety perspective. ChemSusChem 2011; 4: 905-911.

16. Kayat J, Gajbhiye V, Tekade RK, et al. Pulmonary toxicity of carbon nanotubes: A systematic report. Nanomedicine: Nanotechnol Biol Med 2011; 7: 40-49.
17. Liu J-H, Yang S-T, Wang HF, et al. Advances in biodistribution study and tracing methodology of carbon nanotubes. J Nanosci Nanotechnol 2010; 10: 8469-8481.

18. Deng XY, Jia G, Wang HF, et al. Translocation and fate of multi-walled carbon nanotubes in vivo. Carbon 2007; 45: 1419-1424.

19. Yang S-T, Guo W, Lin Y, et al. Biodistribution of pristine single-walled carbon nanotubes in vivo. J Phys Chem C 2007; 111: 17761-17764.

20. Yang S-T, Wang X, Jia G, et al. Long-term accumulation and low toxicity of single-walled carbon nanotubes in intravenously exposed mice. Toxicol Lett 2008; 181: 182-189.

21. Sayes CM, Liang F, Hudson JL, et al. Functionalization density dependence of single-walled carbon nanotubes cytotoxicity in vitro. Toxicol Lett 2006; 161: 135-142.

22. Schipper ML, Nakayama-Ratchford N, Davis CR, et al. A pilot toxicology study of single-walled carbon nanotubes in a small sample of mice. Nat Nanotechnol 2008; 3: 216-221.

23. Wang HF, Wang J, Deng XY, et al. Biodistribution of carbon single-wall carbon nanotubes in mice. J. Nanosci Nanotechnol 2004; 4: 1019-1024.

24. Liu Z, Cai W, He L, et al. In vivo biodistribution and highly efficient tumour targeting of carbon nanotubes in mice. Nat Nanotechnol 2007; 2: 47-52.

25. Cherukuri P, Gannon CJ, Leeuw TK, et al. Mammalian pharmacokinetics of carbon nanotubes using intrinsic near-infrared fluorescence. Proc Natl Acad Sci USA 2006; 103: 18882-18886.

26. Deng XY, Yang S-T, Nie HY, et al. A generally adoptable radiotracing method for tracking carbon nanotubes in animals. Nanotechnology 2008; 19: 075101.

27. Lacerda L, Ali-Boucetta H, Herrero M, et al. Tissue histology and physiology following intravenous administration of different types functionalized multiwalled carbon nanotubes. Nanomedicine 2008; 3: 149-161.

28. Yang F, Hu J, Yang D, et al. Pilot study of targeting magnetic carbon nanotubes to lymph nodes. Nanomedicine 2009; 4: 317-330.

29. Gannon CJ, Cherukuri P, Yakobson BI, et al. Carbon nanotube-enhanced thermal destruction of cancer cells in a noninvasive radiofrequency field. Cancer 2007; 110: 2654-2665.

30. Owens III DE, Peppas NA. Opsonization, biodistribution, and pharmacokinetics of polymeric nanoparticles. Int J Pharm 2006; 300: 93-102.

31. Liu Z, Davis C, Cai W, et al. Circulation and long-term fate of functionalized, biocompatible single-walled carbon nanotubes in mice probed by Raman spectroscopy. Proc Natl Acad Sci USA 2008; 105: 1410-1415.

32. Prencipe G, Tabakman SM, Welsher K, et al. PEG branched polymer for functionalization of nanomaterials with ultralong blood circulation. J Am Chem Soc 2009; 131: 4783-4787.

33. Liu X, Tao H, Yang K, et al. Optimization of surface chemistry on single-walled carbon nanotubes for in vivo photothermal ablation of tumors. Biomaterials 2011; 32: 144-151.

34. de la Zerda A, Zavaleta C, Keren S, et al. Carbon nanotubes as photoacoustic molecular imaging agents in living mice. Nat Nanotechol 2008; 3: 557-562.

35. Yang S-T, Fernando KAS, Liu J-H, et al. Covalently PEGylated carbon nanotubes with stealth character in vivo. Small 2008; 4: 940-944.

36. Huang W, Fernando S, Allard LF, et al. Solubilization of single-walled carbon nanotubes with diamine-terminated oligomeric poly(ethylene glycol) in different functionalization reactions. Nano Lett 2003; 3: 565-568.

37. Lay CL, Liu H, Tan H, et al. Delivery of paclitaxel by physically loading onto poly(ethylene glycol) (PEG)-graft-carbon nanotubes for potent cancer therapeutics. Nanotechnology 2010; 21: 065101. 
38. Cheng J, Meziani MJ, Sun Y-P, et al. Poly(ethylene glycol)-conjugated multi-walled carbon nanotubes as an efficient drug carrier for overcoming multidrug resistance. Toxicol Appl Pharmacol 2011; 250: 184-193.

39. Bhirde AA, Patel S, Sousa AA, et al. Distribution and clearance of PEG-single-walled carbon nanotube cancer drug delivery vehicles in mice. Nanomedicine 2010; 5: 1535-1546.

40. Tasis D, Tagmatarchis N, Bianco A, et al. Chemistry of carbon nanotubes. Chem Rev 2006; 106: 1105-1136.

41. Singh R, Pantarotto D, Lacerda L, et al. Tissue biodistribution and blood clearance rates of intravenously administered carbon nanotube radiotracers. Proc Natl Acad Sci USA 2006; 103: 3357-3362.

42. McDevitt MR, Chattopadhyay D, Jaggi JS, et al. PET imaging of soluble Yttrium-86-labeled carbon nanotubes in mice. PloS ONE 2007; 9: e907.

43. McDevitt MR, Chattopadhyay D, Kappel BJ, et al. Tumor targeting with antibody-functionalized, radiolabeled carbon nanotubes. J Nucl Med 2007; 48: 1180-1189.

44. Pantarotto D, Briand JP, Prato M, et al. Translocation of bioactive peptides across cell membranes by carbon nanotubes. Chem Commun 2004; 1: 16-17.

45. Prato M, Kostarelos K, Bianco A. Functionalized carbon nanotubes in drug design and discovery. Acc Chem Res 2008; 41: 60-68.

46. Podesta JE, Al-Jamal KT, Herrero MA, et al. Antitumor activity and prolonged survival by carbon-nanotube-mediated therapeutic siRNA silencing in a human lung xenograft model. Small 2009; 5: 1176-1185.

47. Al-Jamal KT, Gherardini L, Bardi G, et al. Functional motor recovery from brain ischemic insult by carbon nanotube-mediated siRNA silencing. Proc Natl Acad Sci USA 2011; 108: 10952-10957.

48. Wang J, Deng XY, Yang S-T, et al. Rapid translocation and pharmacokinetics of hydroxylated single-walled carbon nanotubes in mice. Nanotoxicology 2008; 2: 28-32.

49. Liang G, Zhang T, Liu R, et al. Preparation and biodistribution of tyrosine modified multiwall carbon nanotubes. J Nanosci Nanotechnol 2010; 10: 8508-8515.

50. Allen BL, Kichambare PD, Gou P, et al. Biodegradation of single-walled carbon nanotubes through enzymatic catalysis. Nano Lett 2008; 8: 3899-3903.

51. Kagan VE, Konduru NE, Feng W, et al. Carbon nanotubes degraded by neutrophil myeloperoxidase induce less pulmonary inflammation. Nat Nanotechnol 2010; 5: 354-359.

52. Roberts AP, Mount AS, Seda B, et al. In vivo biomodification of lipid-coated carbon nanotubes by Daphnia magna. Environ Sci Technol 2007; 41: 3025-3029.

53. Yang S-T, Wang HF, Meziani MJ, et al. Biodefunctionalization of functionalized single-walled carbon nanotubes in mice. Biomacromolecules 2009; 10: 2009-2012.

54. Worle-Knirsch JM, Pulskamp K, Krug HF. Oops they did it again! Carbon nanotubes hoax scientists in viability assays. Nano Lett 2006; 6: 1261-1268.

55. Ji Z, Zhang D, Li L, et al. The hepatotoxicity of multi-walled carbon nanotubes in mice. Nanotechnology 2009; 20: 445101.

56. Qu G, Bai Y, Zhang Y, et al. The effect of multiwalled carbon nanotube agglomeration on their accumulation in and damage to organs in mice. Carbon 2009; 47: 2060-2069.

57. Bai Y, Zhang Y, Zhang J, et al. Repeated administrations of carbon nanotubes in male mice cause reversible testis damage without affecting fertility. Nat Nanotechnol 2010; 5: 683-689.

58. Zhang D, Deng XY, Ji ZF, et al. Long-term hepatotoxicity of polyethylene-glycol functionalized multi-walled carbon nanotubes in mice. Nanotechnology 2010; 21: 175101.

59. Folkmann JK, Risom L, Jacobsen NR, et al. Oxidatively damaged DNA in rats exposed by oral gavage to $\mathrm{C} 60$ fullerenes and single-walled carbon nanotubes. Environ Health Perspect 2009; 117: 703-708

60. Yang Z, Zhang Y, Yang Y, et al. Pharmacological and toxicological target organelles and safe use of single-walled carbon nanotubes as drug carriers in treating Alzheimer disease. Nanomedicine: Nanotechnol Biol Med 2010; 6: 427-441.

61. Bellucci S, Chiaretti M, Cucina A, et al. Multiwalled carbon nanotube buckypaper: Toxicology and biological effects in vitro and in vivo. Nanomedicine 2009; 4: 531-540.

62. Fraczek A, Menaszek E, Paluszkiewicz C, et al. Comparative in vivo biocompatibility study of single and multi-wall carbon nanotubes. Acta Biomater 2008; 4: 1593-1602.

63. Poland CA, Duffin R, Kinloch I, et al. Carbon nanotubes introduced into the abdominal cavity of mice show asbestoslike pathogenicity in a pilot study. Nat Nanotechnol 2008; 3: $423-428$ 